\title{
APROXIMAÇÕES PARA ENÉSIMO NÚMERO PRIMO
}

\section{ARTIGO ORIGINAL}

OLIVEIRA, Miguel Araújo ${ }^{1}$

OLIVEIRA, Miguel Araújo. Aproximações para Enésimo Número Primo. Revista Científica Multidisciplinar Núcleo do Conhecimento. Ano 05, Ed. 12, Vol. 06, pp. 6472. Dezembro de 2020. ISSN: 2448-0959, Link de acesso: https://www.nucleodoconhecimento.com.br/matematica/enesimo-numero

\section{RESUMO}

Este artigo tem como principal objetivo apresentar uma fórmula algorítmica para calcular o enésimo número primo. Tal fórmula é essencial para partirmos do estado inicial para algum algoritmo mais desenvolvido no futuro. Grandes matemáticos deixaram suas pequenas e grandes descobertas, algumas podendo servir de suporte para a fórmula que será apresentada neste material. Futuramente, esse material pode ser usado para o progresso nos estudos relacionados à teoria dos números podendo enfim, concluirmos uma fórmula fechada para o termo primo geral. Cada contribuição é importante. Logo, todos os pequenos avanços nos fazem chegar mais próximos daquilo que realmente queremos alcançar. Dada a fórmula presente neste artigo, é de suma importância o total compreendimento para que futuros matemáticos possam tomar como inspiração do desenvolvimento possivelmente precoce após essa descoberta. Atribuído de todo o conhecimento sobre a definição de números primos, esse trabalho apresentará uma demonstração da fórmula podendo facilmente ser interpretada pelo leitor.

Palavras-Chave: Fórmula, enésimo termo, número primo.

\footnotetext{
${ }^{1}$ Estudante de Licenciatura em Matemática no Centro Universitário Leonardo da Vinci, Técnico em Auxiliar administrativo pelo Instituto Federal do Pará. Vínculo com Centro Universitário Leonardo da Vinci.
} 


\section{INTRODUÇÃO}

As sequências possuem padrões definidos. Alguns padrões são difíceis de encontrar, como por exemplo: 2, 8,666.., 10,888.., 11,6296296296.., 11,8765432099.., e assim por diante. O segredo por trás dessa sequência é:

\section{$\frac{a 1}{3}+8$}

onde a1 é o primeiro termo da sequência.

$2 / 3+8=8,666 \ldots, 8,666 \ldots / 3+8=10,888 \ldots$ e assim por diante

A sequência dos números naturais obedece uma regra simples. $(1,2,3,4,5,6,7,8$, $9,10,11,12, \ldots$ ). Observe que essa sequência aumenta de 1 em 1 conforme prossegue.

\section{$a 1+1$}

Veja que a equação nesse dois exemplos funciona para todos os termos da sequência se porventura funcionar para o primeiro. Verificando que a sequência é $(1,2,3,4,5$, $6,7,8,9,10,11,12, \ldots)$ vemos que, $a 1=1, \operatorname{logo} 1+1=2$. Considerando 2 como a1, temos $2+1=3$, e assim por diante. Nesses casos é muito simples definirmos o modelo que rege a sequência. Mas, se tratando de números primos o segredo é quase impossível de ser desvendado.

\section{APROXIMAÇÕES PARA SEQUÊNCIAS SIMPLES}

A sequência dos números pares e ímpares é bem interessante, pois as duas consiste na soma de 2 números em relação aos 2 primeiros. Assim como visto no exemplo anterior, podemos modificar o modo com que encontramos os valores da sequência. Exemplo: 
Os números pares; $(0,2,4,6,8,10,12, \ldots)$, observe que a regra por trás dessa sequência é:

\section{$a 1+2$}

pois, se a1 for 0 , então $0+2=2$, dado um novo a1 $=2$, temos $2+2=4$, e assim por diante. Mas, observe que temos outra forma para determinar os elementos dessa sequência:

\section{$a 1+59 / 30$}

dessa forma, usando as regras de arredondamento, podemos determinar os valores pares da sequência.

a1 $+51 / 30$ com a1 $=0$, temos $0+59 / 30=1,9666 \ldots$, arredondando 1,9666 . para inteiro, temos 2. Dado a1 $=2$, temos $2+59 / 30=3,9666 \ldots$, arredondando 3,9666... para inteiro, temos 4. Esse algorítmico serve para definir qualquer sucessor par, como por exemplo o número par depois de 12563456. Dado a1 = 12563456, temos $12563456+59 / 30=12563457,9666 \ldots$, arredondando $12563457,9666 \ldots$ para inteiro, temos 12563458.

\section{TERMO GERAL DE UMA PROGRESSÃO ARITMÉTICA}

Com base no que já foi apresentado até aqui, podemos também determinar o enésimo termo de uma sequência. Dada a sequência dos números pares a seguir:

$0,2,4,6,8,10,12, \ldots$

sabemos que o primeiro termo dessa sequência é 0 , o segundo termo é 2 , o terceiro termo é 4, o quarto termo é 6 , e assim por diante. Mas, qual a regra por trás disso? Como podemos saber qual o quadragésimo oitavo termo dessa sequência sem ficar somando de 2 em 2 até chegarmos na resposta? 


\section{$2 n$}

onde $\mathrm{n}$ é a posição do termo que queremos encontrar, nesse caso o $48^{\circ}$ é $\mathrm{n}$ $=48$, então 2 × 48 = 96, então 96 é quadragésimo oitavo termo da sequência dos números pares.

Por conseguinte, as sequências cuja possuem uma razão $\neq 2$, temos a seguinte fórmula criada por Carl Friedrich Gauss (1777-1885).

\section{$a n=a 1+(n-1) * r$}

onde an é termo geral a ser descoberto, a1 é o primeiro termo da sequência, $\mathrm{n}$ é a posição do termo geral e r a ordem de distribuição da sequência conhecida com razão. Vamos a um exemplo:

Dada a sequência $(0,3,6,9,12,15,18,21, \ldots)$, sabemos que o primeiro termo é 0 , o segundo termo é 3, o terceiro termo é 6 e logo percebemos que a ordem de distribuição é de 3 em 3, sabendo esses dados qual seria o milésimo sexto termo dessa sequência?

$a n=a 1+(n-1) \cdot r$

an $=0+(1006-1) \cdot 3$

an $=0+(1005) \cdot 3$

an $=0+3015$

an $=3015$

Concluímos que, 3015 é o $1006^{\circ}$ termo dessa sequência. No entanto, há sequências praticamente impossíveis de determinarmos sua razão, tornando muito difícil encontrar seu termo geral ou enésimo valor. Como exemplo temos a sequência dos números primos. 


\section{NÚMEROS PRIMOS}

Os números primos são os átomos da Matemática, quando um número não é primo ele é composto por primos. Há casos quando um número não é nem primo e nem composto, como é caso dos números binários. Toda essa relação tem uma sintonia fina matemática por trás da aura do Universo. Cabe aos matemáticos descobrirem o que regem tais leis e determinar suas regras para fins evolutivos em espécie. Sabemos que os números primos estão aí, mas o que não sabemos é qual fórmula gera números primos. O principal intuito desse artigo é apresentar um algorítmico formular que consiste no encontro do termo geral primo.

Observando a sequência a seguir, vemos a beleza como que esses números se comportam:

\section{$2,3,5,7,11,13,17,19,23, \ldots$}

observe que, é praticamente impossível descobrir sua ordem de propagação. Veja que a razão entre os dois primeiros termos é 1, já a razão entre o quarto e quinto é 4 . É evidente que a razão é particular em cada um dos casos, logo não podemos usar a fórmula de Gauss para determinar o termo geral dessa sequência.

\section{ENÉSIMO TERMO PRIMO}

A fórmula aproximada para a sequência dos números primos que será apresentada agora, é relativa ao modelo usado na "aproximação para sequências simples". No entanto temos

$$
\begin{array}{r}
w=\left\{k-\left[k^{0,6348}\right]+\sqrt{5}\right\} \\
\text { onde } k=\left\{\left[(n-1) \pi+2\left(\sqrt[5]{n^{6}}\right)-\sqrt{0,3}[(n-1) \pi+2]\right]\right\} .
\end{array}
$$


Chamaremos "piso de w" a parte inteira de w, usando o símbolo $\mathcal{P}$. Portanto, o termo geral da sequência de números primos fica assim

$$
a n=\mathcal{P}(w) \pi^{0,9595} / 3
$$

sabendo que 0 arredondamento de $\mathcal{P}(\mathrm{w})$ é par, o enésimo primo será o arredondamento de $\mathcal{P}(\mathrm{w})$, mais 1 .

Veja este exemplo para $n=8$ :

$$
\begin{aligned}
& k=\left\{\left[(8-1) \pi+2\left(\sqrt[5]{8^{6}}\right)-\sqrt{0,3}[(8-1) \pi+2]\right]\right\} \Rightarrow \mathrm{k}=33,1021203842 \ldots \\
& \mathrm{w}=\left\{33,1021203842 \ldots-\left[33,1021203842^{\wedge} \sqrt{ } 0,6348\right]+\sqrt{ } 5\right\} \Rightarrow \mathrm{w}=19,0851318565 \\
& \text { an }=19 \pi^{\wedge} 0,9595 \ldots / 3 \Rightarrow 18,9953652647 \\
& \text { an }=18,9953652647 \text { arredondando para inteiro, temos an }=19 .
\end{aligned}
$$

Lembre-se que, relacionado a sequência de números pares, estamos bem próximos de descobrirmos um padrão para a sequência dos primos. Às vezes o valor de an não é tão próximo do valor, às vezes, conseguimos gerar primos de outras casas e por vezes a sua parte inteira é o primo em si. O mais lindo nisso tudo é que com essa visão podemos futuramente desvendar a fórmula fechada para números primos. Dentre alguns dos exemplos que pode acontecer com o valor de an citado temos um exemplo simples. Usando essa fórmula de aproximação para sequências, vamos calcular o quinto e milésimo número primo. Assim, temos

$$
\begin{aligned}
& \mathrm{n}=5 \\
& k=\left\{\left[(5-1) \pi+2\left(\sqrt[5]{5^{6}}\right)-\sqrt{0,3}[(5-1) \pi+2]\right]\right\} \Rightarrow \mathrm{k}=18,3853374625 \ldots \\
& \mathrm{w}=\left\{18,3853374625 \ldots-\left[18,3853374625^{\wedge} \sqrt{0}, 6348\right]+\sqrt{ } 5\right\} \Rightarrow \mathrm{w}=10,4481635634 \ldots
\end{aligned}
$$


an $=10 \pi^{\wedge} 0,9595 \ldots / 3 \Rightarrow 9,9975606656$ arredondando para inteiro temos an $=10$. Lembre-se que, se arredondamento de $\mathcal{P}(\mathrm{w})$ for par, então o valor de an=10+1. Logo, concluímos nesse exemplo que, o quinto número primo é 11.

Para $\mathrm{n}=1000$ usamos o mesmo processo com as mesmas regras e corolários. No entanto, é de suma importância fazermos os cálculos detalhadamente para não concluirmos o valor do $\mathrm{n}$ ésimo primo errado quando se trata de termos gerais grandes como vamos verificar no próximo:

$$
\begin{aligned}
& \mathrm{n}=1000 \\
& k=\left\{\left[(1000-1) \pi+2\left(\sqrt[5]{1000^{6}}\right)-\sqrt{0,3}[(1000-1) \pi+2]\right]\right\} \Rightarrow \mathrm{k}=9380,4985851904 \ldots \\
& \mathrm{w}=\{9380,4985851904 \ldots-[9380,4985851904 \wedge \sqrt{ } 0,6348]+\sqrt{ } 5\} \Rightarrow \mathrm{w}=7921,0803361367 \ldots \\
& \text { an }=7921 \pi 0,9595 \ldots / 3 \Rightarrow 7919,0678032296 \text { arredondando para inteiro, temos an }= \\
& 7919 .
\end{aligned}
$$

\section{METODOLOGIA}

Usa-se o maior número de dados possíveis para podermos definir um padrão. No entanto, o padrão real é quase que impossível. Porém, com pesquisas e com muito esforço conseguimos chegar ainda mais perto no que desejamos.

Verificamos que existem diversas fórmulas que nos mostram a aproximação para números primos. Todas elas em algum dia serão realmente úteis. Dentre estas fórmulas, temos 
1. FÓRMULA DE APROXIMAÇÃO PARA ENÉSIMO PRIMO ASSÍNTOTA.

$$
p_{n}=\left\lfloor 1-\frac{1}{\log 2} \log \left(-\frac{1}{2}+\sum_{d \mid P_{n-1}} \frac{\mu(d)}{2^{d}-1}\right)\right.
$$

Fonte: http://www.mat.puc-rio.br/ nicolau/papers/mersenne/node18.html.

Entendendo e absorvendo a aura de todas essas fórmulas, podemos criar uma nova, ainda mais próxima, para desvendar esse mistério. Contudo, a fórmula apresentada neste é graficamente, um pequeno avanço na compreensão dos números primos.

\section{RESULTADOS E DISCUSSÃO}

Este artigo apresentou uma forma de calcular o enésimo número primo. Tal forma é essencial para partirmos do estado inicial para algum algoritmo mais desenvolvido no futuro. Grandes matemáticos deixaram suas pequenas e grandes descobertas, algumas podendo servir de suporte para a fórmula que fora apresentada neste material. Futuramente, esse material pode ser usado para o progresso nos estudos relacionados à teoria dos números podendo enfim, concluirmos uma fórmula fechada para o termo primo geral. Cada contribuição é importante. Logo, todos os pequenos avanços nos fazem chegar mais próximos daquilo que realmente queremos alcançar.

Observe que, em nosso primeiro exemplo dos números pares o algorítmico que foi apresentado pode nos dar com precisão o número par, o que falta é descobrir o modelo por trás da sequência dos primos. Acredito que o modelo apresentado neste artigo pode ajudar no avanço da compreensão do real mistério que envolve os números primos.

Dada a fórmula presente neste artigo, é de suma importância o total compreendimento para que futuros matemáticos possam tomar como inspiração do desenvolvimento possivelmente precoce após essa descoberta. Atribuído de todo o conhecimento 
sobre a definição de números primos, esse trabalho apresentará uma demonstração da fórmula podendo facilmente ser interpretada pelo leitor.

\section{DEMONSTRAÇÃO PARA PARTE INTEIRA DE $\mathcal{P}(\mathrm{W})$}

Dado $n=1$, temos an $=2$. Se o piso de $[0,904554885-(0,904554885 \sqrt{0,6348})+\sqrt{5}]=2$ quando $n$ vale 1 , então $n \in I R$. Logo a imagem de $f(k)=\{k-[k \sqrt{0}, 6348]+\sqrt{5}\}$ tem parte inteira um número primo, $\forall \mathrm{n} \in \mathrm{IP}$.

\section{FUNDAMENTAÇÃO TEÓRICA E CONSIDERAÇÕES FINAIS}

Sabemos que os números primos são infinitos e que qualquer descoberta voltada para um avanço significativo relativo aos números primos é de suma importância e gera prêmios e etc. No entanto, o principal foco desse artigo é avançar milimetricamente nesse campo contribuindo ativamente para sua compreensão assim como foi usado exemplo dos números pares a1+59/30.

Existe uma fórmula assintótica para encontrarmos os primos, com base nessa fórmula foi realizada alguns estudos no passado para entender o comportamento gráfico dos números primos. Um de seus principais mistérios é seu comportamento gráfico.

2. FUNÇÃO $\pi(x)$, PARA $X \in$

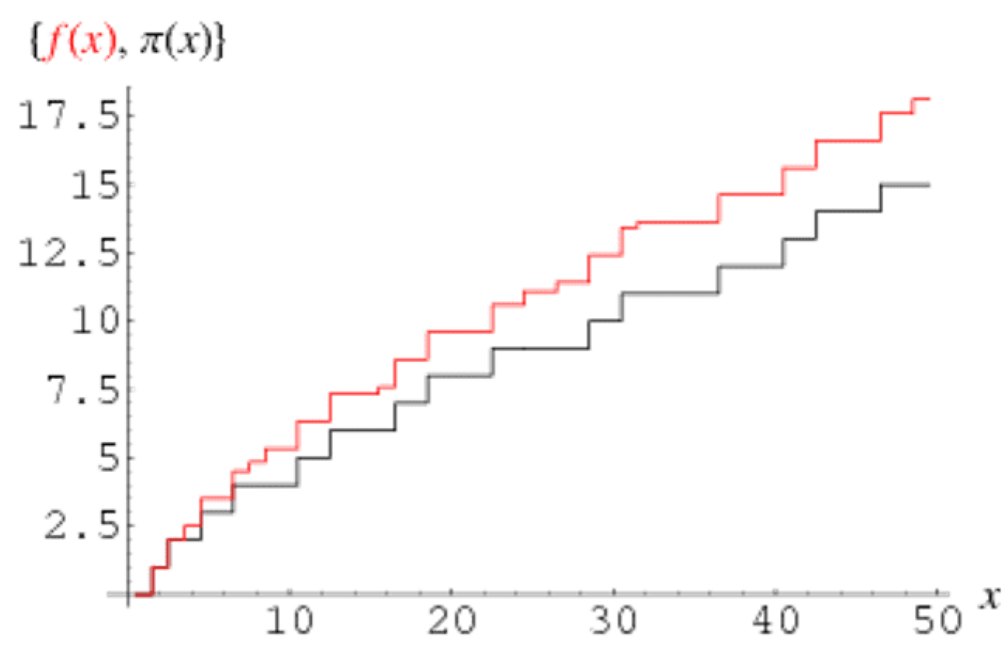

Fonte: Pesquisa no Google[2] 
Com base nos conhecimentos obtidos por Bernhard Riemann (1826-1886), conseguimos entender com uma baixa precisão o comportamento dos números primos graças a sua descoberta. Com isso, deu-se mais um avanço significativo nesta área da Matemática. Esse conceito gráfico foi a fonte de inspiração para a elaboração desse artigo, pois sem os conhecimento obtidos por Bernhard Riemann não seria possível chegar aonde chegamos. No entanto, o que Riemann não conseguiu foi dar continuidade a fórmula para calcular o termo geral não composto. Contudo, essa tarefa fica para as próximas gerações pois Riemann já deixou sua contribuição.

\section{REFERÊNCIAS}

FÓRMULAS PARA PRIMOS E TESTE DE PRIMALIDADE: Disponível em: $<$ http://www.mat.puc-rio.br/ nicolau/papers/mersenne/node18.html>.

FUNÇÃO PARTE INTEIRA: Disponível em: <https://olimpedia.fandom.com/ptbr/wiki/Fun\%C3\%A7\%C3\%A3o_Parte_Inteira\#: :text=A\%20parte\%20inteira\%20de \%20um,com\%20\%22arredondar\%20para\%20baixo\%22.>.

FUNÇÃO DE CONTAGEM: Disponível em: <https://www.google.com/url?sa=i\&url=https\%3A\%2F\%2Fmathworld.wolfram.com\%2 FRiemannPrimeCountingFunction.html\&psig=AOvVaw0Wicgsmc2kX-

YQ6vKBBYIH\&ust=1602798217788000\&source=images\&cd=vfe\&ved=0CA0QjhxqF woTCPC8g6j5iOwCFQAAAAAdAAAAABAJ>.

NÚMERO PRIMO. In: WIKIPÉDIA: a enciclopédia livre. Wikipédia, 2020. Disponível em: <https://pt.wikipedia.org/wiki/N\%C3\%BAmero_primo>.

SÓ MATEMÁTICA: "Progressões" em Só Matemática. Virtuous Tecnologia da Informação, 1998-2020. Consultado em 14/10/2020 às 13:58. Disponível na Internet em: <https://www.somatematica.com.br/emedio/pa/pa3.php>.

BIOGRAFIA

DE

RIEMANN:

Disponível

em:

$<$ https://pt.wikipedia.org/wiki/Bernhard_Riemann>. 


\section{APÊNDICE - REFERÊNCIA DE NOTA DE RODAPÉ}

2.

Disponível

em: https://www.google.com/url?sa=i\&url=https\%3A\%2F\%2Fmathworld.wolfram.co m\%2FRiemannPrimeCountingFunction.html\&psig=AOvVaw0Wicgsmc2kX-

YQ6vKBBYIH\&ust=1602798217788000\&source=images\&cd=vfe\&ved=0CA0QjhxqF woTCPC8g6j5iOwCFQAAAAAdAAAAABAJ

Enviado: Outubro, 2020.

Aprovado: Novembro, 2020. 